\title{
The influence of endogenous and exogenous sex hormones on systemic lupus erythematosus in pre- and postmenopausal women
}

\author{
Bogna Grygiel-Górniak, Mariusz Jacek Puszczewicz \\ Katedra i Klinika Reumatologii i Chorób Wewnętrznych, Uniwersytet Medyczny im. Karola Marcinkowskiego w Poznaniu
}

\begin{abstract}
Systemic lupus erythematosus (SLE or lupus) is a chronic inflammatory disease that occurs mainly in women. Typically, symptoms appear within the first few years of adolescence, but currently an increase can be observed in the percentage of postmenopausal women with this condition. This is possibly due to the sophisticated treatment of the disease, which significantly improves the survival curve and prognosis. Genetic and environmental factors are involved in the development of SLE. Both regulation of the immune system and the activity of this disease are influenced by a variety of hormones, including: 17 $\beta$-estradiol, testosterone, prolactin, progesterone and dehydroepiandrosterone (DHEA). Early menarche, menstrual cyclicity, the total number of years characterized by ovulatory cycles and early menopause are correlated with the development of SLE.

Because of the health risks, attempts are increasingly being made to evaluate the impact of exogenous hormones (especially those applied exogenously) on the course of SLE. In particular, the role of estrogens is being highlighted, either endo- or exogenous, including oral contraceptives (OC), therapy used in the treatment of infertility, and hormonal replacement therapy (HRT). The purpose of this manuscript is the revision of the literature concerning the impact of both endo- and exogenous estrogens on the development of lupus, inducement of flares and any possible complications.
\end{abstract}

Key words: endogenous and exogenous estrogens, systemic lupus erythematosus, hormonal therapy.

\section{Introduction}

The influence of estrogens on the development of the systemic lupus erythematosus (SLE) remains unclear, because conflicting data are present in the literature. Some of them underline the negative influence of these hormones on the immune system [1-6], especially in patients with some genetic predisposition $[7,8]$, while others show the positive influence on health $[9,10]$.

The aetiology of SLE (as with many other autoimmune diseases) is unknown, but it seems that environmental factors initiate its development in genetically predisposed individuals $[1,2,11]$. Considering the fact that SLE is present mainly in women $(80-90 \%$ of patients), it is assumed that sex hormones play an important role in the pathogenesis of this disease [1]. Moreover, both endo- and exogenous estrogens can increase the risk of SLE flares and complications (e.g. venous thrombosis) [2, 3, 12-14].

Since women frequently use pharmacological supplementation of estrogens in different periods of life, many studies have attempted to assess their impact on health. The effect of estrogens is particularly important in women with lupus, because of the increased risk as- sociated with hormonal therapy itself as well as with the course of SLE $[1,2]$. It has been proved that steroid hormones such as $17 \beta$-estradiol, testosterone, prolactin, progesterone and dehydroepiandrosterone (DHEA) influence immune system regulation $[3,4]$ and the activity of SLE $[5,6]$. Grimaldi et al. found that estrogens play an important role in B cell maturation, selection and activation and, thus, can potentially weaken the immune system [3]. Meta-analysis of the effect of the hormonal system on SLE has shown significantly lower serum concentrations of androgens (testosterone and DHEA) and higher concentrations of estradiol and prolactin in female patients when compared to control groups, i.e. healthy individuals $[2,3,5,7]$.

\section{Hormonal influence on the development of systemic lupus erythematosus genetic factors}

Since hormonal changes may be genetically determined, the polymorphism of estrogen receptor $\alpha$ (ER, codon 594) has been analyzed in the pathogenesis of SLE. In the Greek population, the polymorphism of this recep- 
tor is associated with the development of the disease at a younger age, and the tendency to skin changes, lesions in the oral cavity and inflammation of the mucous membranes [8]. Furthermore, a study on Swedish women revealed the coexistence of rare Pvull $C$ and $X b a l ~ G$ alleles with a later onset of SLE, skin changes, and a milder course of the disease [7]. These studies showed that both polymorphisms are associated with the age of menarche and menopause, and with greater susceptibility to estrogen influence $[8,12]$. This provided the background to enable the conclusion to be drawn that there is a relationship between genetic predisposition and the influence of estrogens and their receptors and the development of SLE. Consequently, genetic studies have enabled the formation of the thesis that in the development of SLE different genes are involved, the activity of which depends on the influence of endogenous and exogenous estrogens, and thus different phenotypes of lupus are observed $[7,8]$.

\section{Hormonal changes in puberty and the risk of systemic lupus erythematosus}

Hormonal changes during puberty are caused by altered endogenous sex hormone secretion, which can influence the onset of clinical manifestations and the course of SLE. It seems that the age of menarche (the first moment of systemic exposure to estrogens) is a risk factor for this disease. Clinical studies have shown a correlation between the first clinical manifestation of SLE and an early ( $\leq 10$ years of age), occasionally late, menarche $[2,13]$. In puberty, hormonal changes are caused by the maturation of the hypothalamicpituitary axis and are associated with the beginning of pulsatile gonadotropin-releasing hormone secretion. An analysis of two large studies - NHS and NHS II (the Nurses' Health Study) - has confirmed the association of early menstruation with the development of SLE [14, 15]. Nevertheless, it should be noted that the first menarche is genetically determined and depends on the race and partially on nutritional status (including body mass index - BMI) and physical activity. It is also related to increased birth weight, body composition (fat and muscle mass) and the growth spurt in early childhood. Early first menarche is also correlated with an increased risk of endometriosis and breast cancer [8].

Not only the age of menarche, but also menstrual cyclicity influence the onset of SLE. Observational studies analysing the hormonal stage in a group of Japanese women showed that irregular menstruation was associated with an increased risk of the development of this disease [2]. In addition, one population study (the Carolina Lupus Study) has demonstrated that both long and short menstrual cycles correlate with the development of SLE [13]. However, Costenbader et al. did not observe any relationship between the frequency of ovulatory cy- cles and the onset of lupus, which suggests that it is not the exposure of endogenous estrogens itself, but rather the time of its occurrence that is associated with a risk of lupus [16]. Moreover, the total number of years with ovulatory cycles have been shown to be an independent risk factor for the development of this disease [13, 16].

Although many studies have shown the increased activity of SLE in pregnancy [17], the number of births is not associated with a higher risk of this disease $[13,17]$. A study by Cooper et al. showed that a long total period of breast-feeding was inversely correlated with the incidence of SLE [13], but other studies have not confirmed such a relationship [16].

\section{Oral contraception and the development of systemic lupus erythematosus}

Even though the influence of exogenous hormones on the development of SLE is widely discussed, there are still many inconsistencies. A randomized study of American and Mexican women showed that the use of oral contraceptives was not associated with an increase in the frequency of lupus flares in women with mild and stable forms of the disease $[13,14]$. Similarly, the SELENA study (Safety of Estrogens in Lupus Erythematosus - National Assessment) revealed that oral contraceptives containing estrogens did not increase the risk of lupus flares [12]. However, many authors have claimed that such hormonal supplementation can stimulate the development of SLE $[1,2]$. A tendency towards an increased incidence of SLE in patients using oral contraceptives was established in a prospective populationbased study - NHS [15]. The dose-dependent effect of exogenous estrogens was not confirmed, but the risk of SLE development was increased over the subsequent ten years, even after discontinuation of oral contraception [16]. Such inconsistencies of hormonal supplementation may result from the characteristics of the study group (such as age, nationality and race), activity and duration of lupus during study, and the type of OC applied. It is noteworthy that estrogens increase the possibility of the development and/or flares of the disease, while androgens have a protective effect (e.g. exogenous progesterone) $[5,6,17]$.

\section{Menopause and its influence on the manifestation of systemic lupus erythematosus}

Systemic lupus erythematosus occurs mainly in young women, but recently an increase in the incidence of this disease has been observed in postmenopausal women. This fact may be explained by therapeutic possibilities which significantly increase the survival curve, improve the prognosis and means that more 
women with SLE survive into their late postmenopausal years [1, 2].

One of the risk factors associated with the development of SLE is an early age of menopause. It has been proved that rapid cessation of the ovarian function may affect the onset of lupus (NHS study) [14]. The other risk factors for SLE include surgical menopause (caused by bilateral ovariectomy), mainly in women who undergo the operation at ages lower than the average age of menopause. On the other hand, it should be emphasized that in women with systemic lupus menopause occurs earlier when compared to healthy women, as was confirmed by NHS and the Carolina Lupus Study $[9,13,14]$.

\section{Hormonal replacement therapy and the risk of systemic lupus erythematosus}

Menopause is associated with an increased risk of osteoporosis and vasomotor disorders. Although hormonal replacement therapy (HRT) is the most effective method for the treatment of vasomotor and urogenital postmenopausal changes, large population-based studies, such as HERS (Heart and Estrogen/Progestin Replacement Study) and WHI (the Women's Health Initiative), have shown an increased health risk after supplementation of HRT $[9,20]$. The HERS study revealed that women with coronary artery disease who used HRT (CEE - conjugated equine estrogens - $0.625 \mathrm{mg}$ and MPA - medroxyprogesterone acetate $-2.5 \mathrm{mg}$ ) had an increased incidence of coronary events (mainly during the first year of therapy), thromboembolism, gallstones and breast cancer, when compared to placebo groups. However, a decreased incidence of colon cancer, endometrium, and a lowered risk of osteoporosis was also observed [9]. Another large multicenter study - WHI (financed by the National Institutes of Health and focusing on women without coronary artery disease) - was prematurely terminated (after 5.6 years of follow-up) due to an increased incidence of breast cancer. Moreover, an enhanced risk of myocardial infarction and venous thromboembolism in the group using HRT was confirmed [20]. Consequently, the results of HERS and the WHI study have changed the approach to the treatment of postmenopausal hormonal insufficiency.

Prospective studies by Sánchez-Guerrero et al., which included two years of observation, revealed that HRT influenced lupus activity in a group of postmenopausal Mexican women in the same way as placebo treatment, irrespective of early or late menopause [10]. However, the risk of SLE flares was slightly increased after HRT implementation, especially when lupus activity was high at the onset of this disease. The frequency of flares was similar in the study and control groups, and the total median time of flares was three months in both groups [10]. It must be underlined that the activity of lupus in this study was low (study group SLEDAI $=3.5 \pm 3.3$ and control SLEDAI = $3.1 \pm 3.4, p=0.57$ ), and this may also impact upon any final conclusions (SLEDAI - Systemic Lupus Erythematosus Disease Activity Index - an indicator of disease activity in lupus; 0 points - inactive disease, $1-5$ points - mild activity, 6-10 points - moderate activity, 11-19 points - high activity, $\geq 20$ points - very high disease activity).

Currently, surveys have not confirmed that lupus flares or the activity of the disease depend on the dose of exogenous estrogen, but it seems that an increased risk of SLE development is maintained for five years after the cessation of HRT [15]. Besides the increased morbidity of lupus associated with postmenopausal HRT [13], a slight increase in the risk of moderate (but not acute) lupus flares is observed (SELENA study) [21]. Similar observations were described by Jungers et al. who used a mixed (two-component) HRT [8]. Randomized studies on American [18] and Mexican women [10] showed an increase in the frequency of lupus flares after one year of the use of HRT [21].

Hormonal replacement therapy also influences the development of SLE complications. It increases threefold the risk of venous thrombosis when compared to placebo groups (SELENA study) [21]. Similar correlations have been noticed in observational [22] and clinical studies [20] concerning healthy women, and in clinical studies regarding patients with lupus [10]. Although venous thrombosis occurs in the general population, an increased incidence of this disease is observed in patients with SLE. In healthy women under 30 years of age the incidence of thrombosis is 0.05 per 1000 person/ year [23], whereas in postmenopausal age this figure reaches 0.08-0.11 per 1000 person/year [22]. In lupus the incidence of thrombosis dramatically increases and it occurs in $10-20 \%$ of patients with this disease [24, 25] reaching a value of 5.1 per 1000 person/year [24]. Statistics show that the total risk of thrombosis taken from the onset of the disease, increases to 51.9 per 1000 person/year [25]. Taking into consideration the influence of exogenous estrogen on the development of thrombotic changes, it must be underlined that the absolute risk for women with lupus using HRT is very high [22].

The increased risk of venous thrombosis in the course of SLE also depends on the presence of antiphospholipid antibodies, cigarette smoking, older age, female gender, lupus activity and the dose of glucocorticosteroids taken [26]. Continuous sequential estrogen-progestin therapy (such as medroxyprogesterone acetate) may increase the proinflammatory effect of estrogens [27]. Therefore, when HRT is implemented, the merits of such therapy should be carefully considered and the risks should be carefully balanced against 
the benefits in subjects with SLE. Unfortunately, there are no studies showing that either alternative methods of administration of estrogens, or lower doses of the hormones compared to standard HRT, can minimize the possibility of lupus onset, its flares and possible complications.

Despite the risks associated with the use of HRT in SLE, it is believed that many women should use such therapy due to their severe symptoms of menopause, which significantly negatively affect their quality of life. Current recommendations suggest that HRT should be used in the lowest possible dose and for the shortest possible time, but the risk of potential complications of such therapy should always be considered [28, 29].

\section{Conclusions}

The role of female sex hormones in the development of SLE is complex and difficult to study due to a relatively low incidence of SLE in the general population. Women with lupus are at an increased risk of early first menarche and menopause, cardiovascular disorders, premature atherosclerosis and thrombosis, osteoporotic fractures, cognitive impairment and reduced quality of life when compared to healthy subjects. Although the clinical studies thus far undertaken do not clearly indicate that the use of oral contraceptives is associated with the development of the disease or flares, such an association is frequently observed during hormonal replacement therapy. It has been proven that exogenous hormones are also involved in the development of thrombosis, the risk of which is increased in the case of lupus. Independently of this, neither the time of menarche nor menopause are risk factors for lupus development. In conclusion, the use of hormonal therapy should be carefully analyzed with careful consideration of the advantages and disadvantages of such treatment in women with SLE.

\section{Disclosure}

Authors report no conflicts of interest.

\section{References}

1. Zandman-Goddard G, Solomon M, Rosman Z, et al. Environment and lupus related diseases. Lupus 2012; 21: 241-250.

2. McMurray RW, May W. Sex hormones and systemic lupus erythematosus: review and meta-analysis. Arthritis Rheum 2003; 48: 2100-2110.

3. Grimaldi CM, Hill L, Xu X, et al. Hormonal modulation of B cell development and repertoire selection. Mol Immunol 2005; 42: 811-820.

4. Vera-Lastra O, Jara LJ, Espinoza LR. Prolactin and autoimmunity. Autoimmun Rev 2002; 1: 360-364.

5. Chang DM, Lan JL, Lin HY, et al. Dehydroepiandrosterone treatment of women with mild-to-moderate systemic lupus erythematosus: a multicenter randomized, double-blind, placebocontrolled trial. Arthritis Rheum 2002; 46: 2924-2927.
6. Petri MA, Lahita RG, van Vollenhoven RF, et al. for the GL701 Lupus Study Group. Effects of prasterone on corticosteroid requirements of women with systemic lupus erythematosus: a double-blind, randomized, placebo-controlled trial. Arthritis Rheum 2002; 46: 1820-1829.

7. Johansson M, Arlestig L, Moller B, et al. Oestrogen receptor alpha gene polymorphisms in systemic lupus erythematosus. Ann Rheum Dis 2005; 64: 1611-1617.

8. Stavrou I, Zois C, loannidis JP, et al. Association of polymorphisms of the oestrogen receptor alpha gene with the age of menarche. Hum Reprod 2002; 17: 1101-1105.

9. Weel AE, Uitterlinden AG, Westendorp IC, et al. Estrogen receptor polymorphism predicts the onset of natural and surgical menopause. J Clin Endocrinol Metab 1999; 84: 3146-3150.

10. Cooper GS, Dooley MA, Treadwell EL, et al. Hormonal and reproductive risk factors for development of systemic lupus erythematosus: results of a population-based, case-control study. Arthritis Rheum 2002; 46: 1830-1839.

11. Jędryka-Góral A. The role of environmental factors in the etiology of systemic lupus erythematosus. Reumatologia 2013; 51: 26-30.

12. Sánchez-Guerrero J, Liang MH, Karlson EW, et al. Postmenopausal estrogen therapy and the risk for developing systemic lupus erythematosus. Ann Intern Med 1995; 122: 430-433.

13. Sánchez-Guerrero J, Karlson EW, Liang MH, et al. Past use of oral contraceptives and the risk of developing systemic lupus erythematosus. Arthritis Rheum 1997; 40: 804-808.

14. Costenbader KH, Feskanich D, Stampfer MJ, et al. Reproductive and menopausal factors and risk of systemic lupus erythematosus in women. Arthritis Rheum 2007; 56: 1251-1262.

15. Petri M. Prospective study of systemic lupus erythematosus pregnancies. Lupus 2004; 13: 688-689.

16. Petri M, Kim MY, Kalunian KC, et al. for the OC-SELENA Trial. Combined oral contraceptives in women with systemic lupus erythematosus. N Engl J Med 2005; 353: 2550-2558.

17. Chang DM, Lan JL, Lin HY, et al. Dehydroepiandrosterone treatment of women with mild-to-moderate systemic lupus erythematosus: a multicenter randomized, double-blind, placebo-controlled trial. Arthritis Rheum 2002; 46: 2924-2927.

18. Hulley S, Grady D, Bush T, et al. the Heart and Estrogen/progestin Replacement Study (HERS) Research Group. Randomized trial of estrogen plus progestin for secondary prevention of coronary heart disease in postmenopausal women. JAMA 1998; 280: 605-613.

19. Rossouw JE, Anderson GL, Prentice RL, et al. Risks and benefits of estrogen plus progestin in healthy postmenopausal women: principal results from the Women's Health Initiative randomized controlled trial. JAMA 2002; 288: 321-333.

20. Sánchez-Guerrero J, González-Pérez M, Durand-Carbajal M, et al. Menopause hormonal therapy in women with systemic lupus erythematosus. Arthritis Rheum 2007; 56: 3070-3079.

21. Buyon JP, Petri MA, Kim MY, et al. The effect of combined estrogen and progesterone hormone replacement therapy on disease activity in systemic lupus erythematosus: a randomized trial. Ann Intern Med 2005; 142: 953-962.

22. Daly E, Vessey MP, Hawkins MM, et al. Risk of venous thromboembolism in users of hormone replacement therapy. Lancet 1996; 348: 977-980.

23. Lowe GD. Venous and arterial thrombosis: epidemiology and risk factors at various ages. Maturitas 2004; 47: 259-263.

24. Somers E, Magder LS, Petri M. Antiphospholipid antibodies and incidence of venous thrombosis in a cohort of patients with systemic lupus erythematosus. J Rheumatol 2002; 29: 2531-2536.

25. Sarabi ZS, Chang E, Bobba R, et al. Incidence rates of arterial and venous thrombosis after diagnosis of systemic lupus erythematosus. Arthritis Rheum 2005; 53: 609-612.

26. Calvo-Alen J, Toloza SM, Fernandez M, et al. for the LUMINA Study Group. Systemic Lupus Erythematosus in a Multiethnic US Cohort (LUMINA). XXV. Smoking, older age, disease activity, lupus anticoagulant, and glucocorticoid dose as risk factors for the occurrence of venous thrombosis in lupus patients. Arthritis Rheum 2005; 52: 2060-2068. 
27. Wakatsuki A, Okatani Y, Ikenoue N, et al. Effect of medroxyprogesterone acetate on vascular inflammatory markers in postmenopausal women receiving estrogen. Circulation 2002; 105: 1436-1439.

28. The North American Menopause Society. Estrogen and progestogen use in peri- and postmenopausal women: March 2007 position statement of The North American Menopause Society. Menopause 2007; 14: 168182.

29. Stachon AJ. Feeling of the selected climacteric symptoms depending on the climacteric phase and type of menopause. Prz Menopauzalny 2013; 17: 315-320. 